\title{
Bioseparation
}

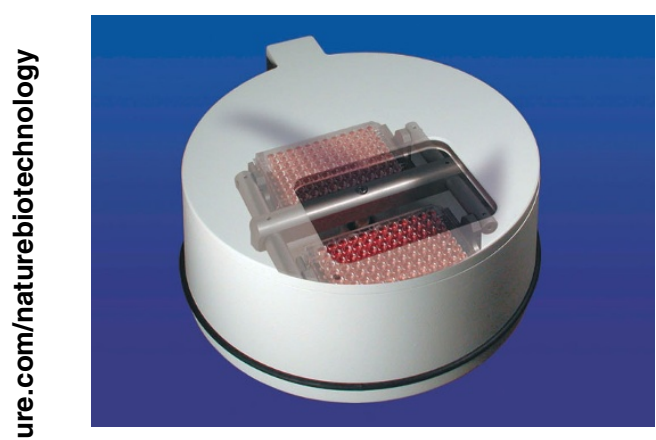

Robot-friendly microcentrifuge

The Ixion microplate centrifuge module is designed for complete robotic integration, with a loading shutter that opens and closes under software control and an automatic homing position to facilitate robotic access. It accommodates one or two microplates, deepwell plates or gel cards and operates quietly, with minimal vibration.

http://www.sias.biz/

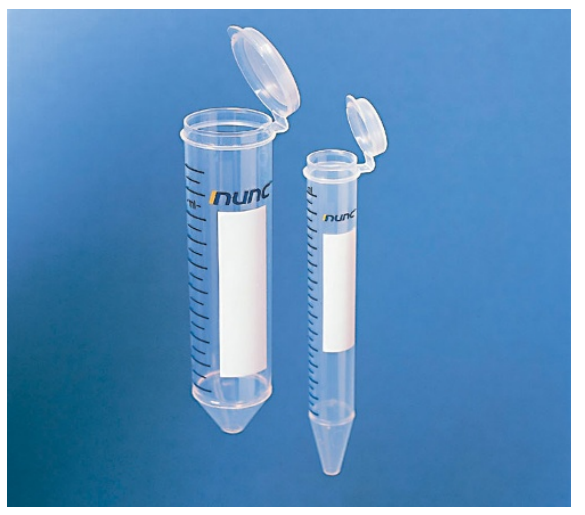

\section{Centrifuge tubes}

EZFlip 15- and 50-ml conical centrifuge tubes, from Nunc, can be used in protein and DNA precipitation, subcellular fractionation, sample storage and cell pelleting of all types. A patented hinged-cap closure system allows for easy, single-handed opening. The tubes are airtight, leak-proof and chemical resistant, and are designed with standard dimensions to be used in most fixed-angle or swing-out rotors. The side of each tube has graduations for easy assessment of sample volume, and a white writing area on the opposite side allows for clear sample identification.

http://www.nuncbrand.com/

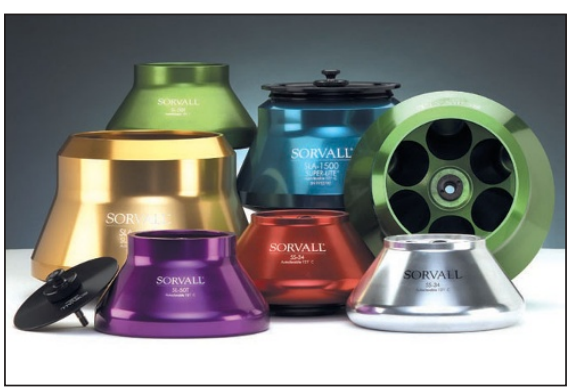

\section{Colored rotors}

Sorvall's ColorTone rotors stand out from standard black centrifuge rotors to enhance laboratory safety and improve rotor inventory management. Thirteen different fixed-angle Superspeed rotors are available for Sorvall RC5 Series, Evolution RC and Super T 21 centrifuges in anodized blue, red, green, purple, gold and silver. Users can enhance laboratory safety by color-designating specific rotors for biohazardous or radioactive applications. Color designation by protocol or sample type can also help prevent cross-contamination. http://www.kendro.com/

\section{Antibodies}

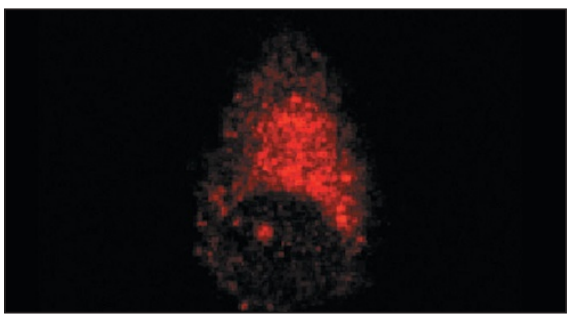

\section{Aph-1a and Aph-1b}

The gamma secretase is a multiprotein complex that catalyzes cleavage of a number of integral membrane proteins, including amyloid precursor protein, Notch, Erb4, E-cadherin and p75 NTR. Aph-1 stabilizes the presenilin holoprotein within the gamma secretase complex, and Covance has introduced highly specific, affinity-purified polyclonal antibodies specific for the $\mathrm{C}$ terminus and loop region of Aph-1a and Aph-1b, the two genes encoding Aph-1. These antibodies are suitable for western blotting and immunostaining. http://www.crpinc.com/

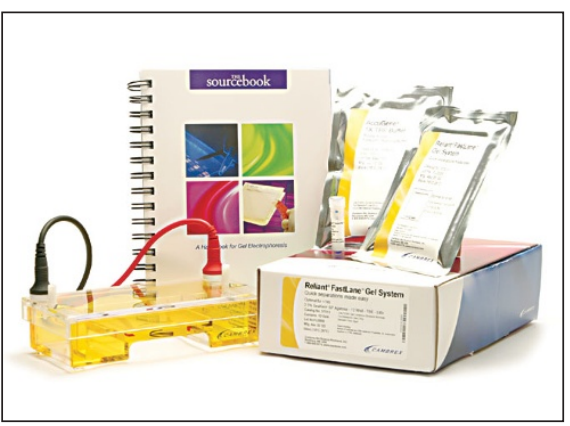

Precast gel system

The Reliant FastLane precast gel system and the Reliant FastLane starter kit, from Cambrex Bio Science Rockland, allows for the quick separation and analysis of PCR products. DNA fragments from $10 \mathrm{bp}$ to $10 \mathrm{~kb}$ can be accurately resolved in as little as $15 \mathrm{~min}$ utes. The system includes a precision-cast agarose gel and an accurately premixed, premeasured separation buffer, both specifically engineered to optimize running performance in the FastLane chamber.

http://www.cambrex.com/

\section{NMDA receptor}

QED Bioscience offers a monoclonal antibody to the glutamate NMDA receptor subunits $3 \mathrm{~A}$ and $3 \mathrm{~B}$. It recognizes both subunits in western blotting and immunohistochemistry. When expressed in Xenopus laevis oocytes, NR3A or NR3B assemble with NR1 to form excitatory glycine receptors that are unaffected by glutamate. http://www.qedbio.com/

\section{siRNA analysis}

Ambion expands its siRNA research offerings with mouse monoclonal antibodies for the detection of GAPDH, c-myc and $\beta$-actin by immunofluorescence. These antibodies are ideal for use in double-labeling experiments with fluorescently labeled siRNA to correlate the uptake of siRNA with the down-regulation of target expression. Antibodies are supplied in solution in $100-\mu \mathrm{g}$ unit sizes. http://www.ambion.com/ 